\title{
Archaea Signal Recognition Particle Shows the Way
}

\author{
Christian Zwieb ${ }^{1}$ and Shakhawat Bhuiyan ${ }^{2}$ \\ ${ }^{1}$ Department of Molecular Biology, University of Texas Health Science Center at Tyler, 11937 US Highway 271, Tyler, \\ TX 75708-3154, USA \\ ${ }^{2}$ Department of Biology, Division of Business and Sciences, Jarvis Christian College, P.O. Box 1470, Hawkins, TX 75765, USA
}

Correspondence should be addressed to Christian Zwieb, zwieb@uthct.edu

Received 15 April 2010; Accepted 14 May 2010

Academic Editor: Jerry Eichler

Copyright $\odot 2010$ C. Zwieb and S. Bhuiyan. This is an open access article distributed under the Creative Commons Attribution License, which permits unrestricted use, distribution, and reproduction in any medium, provided the original work is properly cited.

\begin{abstract}
Archaea SRP is composed of an SRP RNA molecule and two bound proteins named SRP19 and SRP54. Regulated by the binding and hydrolysis of guanosine triphosphates, the RNA-bound SRP54 protein transiently associates not only with the hydrophobic signal sequence as it emerges from the ribosomal exit tunnel, but also interacts with the membrane-associated SRP receptor (FtsY). Comparative analyses of the archaea genomes and their SRP component sequences, combined with structural and biochemical data, support a prominent role of the SRP RNA in the assembly and function of the archaea SRP. The 5e motif, which in eukaryotes binds a 72 kilodalton protein, is preserved in most archaea SRP RNAs despite the lack of an archaea SRP72 homolog. The primary function of the 5e region may be to serve as a hinge, strategically positioned between the small and large SRP domain, allowing the elongated SRP to bind simultaneously to distant ribosomal sites. SRP19, required in eukaryotes for initiating SRP assembly, appears to play a subordinate role in the archaea SRP or may be defunct. The N-terminal A region and a novel C-terminal R region of the archaea SRP receptor (FtsY) are strikingly diverse or absent even among the members of a taxonomic subgroup.
\end{abstract}

\section{Introduction}

Protein sorting fundamentally maintains the identity and function of every cell with participation of the signal recognition particle (SRP). SRP components have been found in nearly all organisms [1]. Except in chloroplasts, SRP is a ribonucleoprotein [2]. The SRP RNA is typically composed of about 300 nucleotide residues and forms a complex with an extraordinarily conserved protein named SRP54 in archaea and eukarya or Ffh (fifty-four homolog) in the bacteria. A $19 \mathrm{kDa}$ protein, SRP19, is present in archaea and eukarya, but absent in the bacteria. Polypeptides which are homologous to the eukaryal SRP9/14 and SRP68/72 heterodimers have not been found in the archaea genome sequences giving rise to an archaea SRP which is dominated by RNA $[3,4]$.

SRP interacts with secretory signal or membrane-anchor sequences upon their emergence from the ribosomal exit tunnel. In vitro and in vivo experiments carried out in eukaryotic protein sorting systems have shown that the SRP delays or blocks the translation of the to-betargeted polypeptides. Translation resumes when the SRPbound ribosome nascent chain complex (RNC) binds to the membrane-associated FtsY (filamentous temperature sensitive $\mathrm{Y}$ ) or, in eukaryotes, the alpha subunit of the $\mathrm{SRP}$ receptor $(\mathrm{SR} \alpha)$. The interaction between SRP54 and the SR increases the affinity of the proteins for guanosine triphosphate, promotes the release of the signal from the SRP, and interjects the signal sequence of the nascent polypeptide into the protein-conducting channel (PCC) of the cell membrane. Translation and protein translocation or membrane insertion take place during ongoing translation (cotranslational translocation), and, upon hydrolysis of two GTP molecules, the SRP returns to its free cytosolic state ready to initiate another protein targeting cycle (Figure 1) [5-9].

Even though archaea membranes differ significantly from the cell membranes of eukaryotes and bacteria with regard to the use of glycerol-ether lipids and isoprenoid side chains $[10,11]$, no obvious adaptations for survival under extreme 


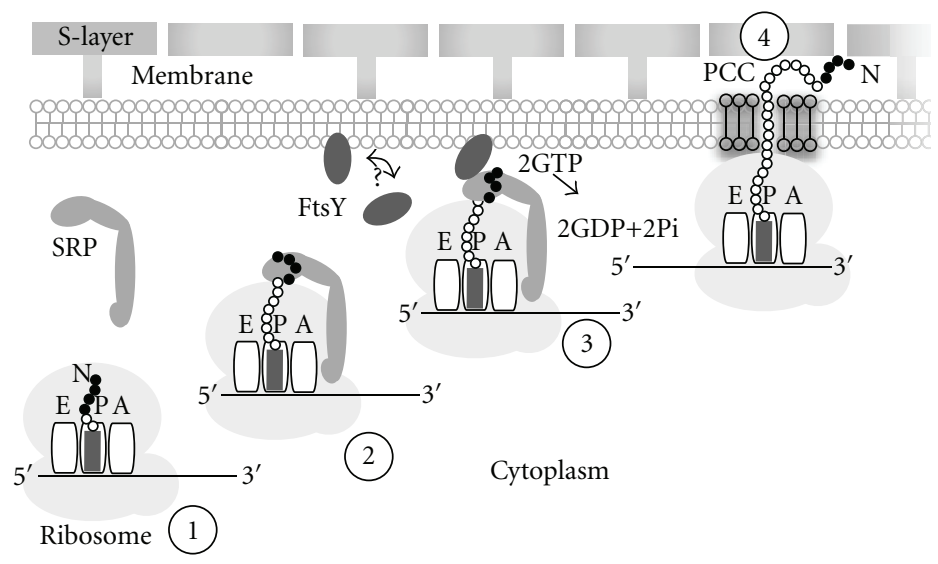

FIGURE 1: Hypothetical steps in the SRP-mediated targeting of archaea proteins. Step 1: A ribosome (gray, with A, P and E tRNA binding sites) in the cytoplasm translates a mRNA molecule (black, $5^{\prime}$ and $3^{\prime}$ ends are indicated) which encodes a N-terminal signal or membraneanchor sequence (black dots). Step 2: As the signal emerges from the large ribosomal subunit, it is recognized by the elongated SRP and further translation may be halted. Step 3: The SRP-bound ribosome nascent chain complex (RNC) binds to free or membrane-associated FtsY (arrow). Step 4: After GTP hydrolysis, SRP has been released, translation resumes, and the targeted protein is threaded through the protein-conducting channel (PCC). The surface (S) layer, present in most archaea, is anchored to a glycerol-ether lipids-containing cell membrane.

conditions are apparent in the SRP components. Like bacteria, archaea contain only one SRP receptor polypeptide, FtsY, a homolog of the eukaryal $\mathrm{SR} \alpha$ subunit. The signal sequences of the archaea and bacteria are interchangeable $[12,13]$, and archaea signal peptidases which remove the signal sequence after protein translocation have been identified [14]. Archaea lack homologs of the bacterial and eukaryal translocation ATPases SecA and Kar2p/BiP. They possess, however, Sec61 (the PCC) and a Tat translocase system [15]. These SRP independent means of protein delivery have been discussed recently $[16,17]$ and will not be reviewed here.

Solving the structures of numerous archaea SRP components and their complexes at high resolution (Table 1) has been crucial for understanding the intricacy of protein targeting in all organisms. Within this structural framework, the increasing number of newly identified archaea genome sequences provides an opportunity to review and discover not only archaea-specific SRP features, but also draw phylogenic distinctions which may pave the way for a better understanding of the function and evolution of every SRP.

\section{Archaeal SRP RNA}

Unlike the bacterial and eukaryal SRP RNAs, their archaea counterparts vary little in shape and size (approximately 300 nucleotide residues). This may be due to relatively slow evolutionary rates as has been observed previously when the relative conservation of archaea protein sequences was investigated [31]. Archaea SRP RNA secondary structures possess extensively base paired regions which form a prominent central helix flanked by a small (or Alu) and a large (or S) domain (Figure 2(a)). Thus, they resemble the secondary structures of the mammalian SRP RNAs. Helices have been assigned numbers from one to eight, and helical section are designated with letters a to $f[32,33]$. The SRP RNAs of most archaea as well as certain bacteria (e.g., Bacilli and Clostridia) pair their terminal regions to form a helix 1 . Helix 7 has been found only in eukaryal SRP RNAs where it is most prominent in some fungi and protozoans [1].

Using the previously described sequence identification procedures and covariation rules (see Methods) we aligned 81 archaea SRP RNA sequences and arranged them according to NCBI's taxonomy [35]. The shared alignment pairing mask allows to deduce phylogenetically supported SRP RNA secondary structures for each of the aligned sequences. With a few exceptions, a sequence corresponds to a known species.

The apical loops of SRP RNA helices 3 and 4 form a tertiary interaction which is well supported by covarying compensatory base changes. The UGUNR sequence motif ( $\mathrm{N}$ is $\mathrm{A}, \mathrm{C}, \mathrm{G}$ or $\mathrm{U}, \mathrm{R}$ is a purine) located between these helices (labeled UGU in Figure 2(a)) is part of a structurally important U-turn. Both features promote the high degree of compactness of the small SRP domain. It remains to be determined how similar the structure of the protein-free small domain of the archaea SRP is to the solved crystal structure of the mammalian Alu domain in complex with the SRP9/14 protein heterodimer [36].

As previously noted and confirmed by mining of the larger collection of archaea SRP RNA sequences, deviations from the UGUNR motif occur in several groups [37]. Conspicuous erosions of the small domain take place in the SRP RNAs of several Desulfurococcales and in Nitrosopumilus maritimus SCM1. Base pairs which typically participate in the formation of helices 1 and 3 are absent in these sequences, while other residues perhaps form an extended helix 4. Due to the relatively small number of available sequences within these subgroups it is not yet possible to conclusively prove or disprove plausible base pairs.

Another hydrogen-bonded tertiary interaction engages two adenosines within the apical tetraloops of helices 6 and 8 
TABLE 1: High-resolution structures of archaeal SRP components. Indicated are the archaea subdomains (Crenarchaeota or Euryarchaeota), species names, components, and methods (X-Ray diffraction of NMR) used for structure determination. The pdb IDs allow easy retrieval of the coordinates [18]. The protein-conducting channel is abbreviated as PCC. Additional nonarchaea SRP high-resolution structures are listed at http://rnp.uthct.edu/rnp/SRPDB/srpstructures.html.

\begin{tabular}{|c|c|c|c|c|c|}
\hline Subdomains & Species & Components & Methods & $\mathrm{pdb}$ & References \\
\hline \multirow[t]{5}{*}{ Crenarchaeota } & Acidianus ambivalens & SRP54NG & X-Ray & 1J8M,1J8Y & [19] \\
\hline & Sulfolobus solfactaricus & SRP54 with helix 8 & X-Ray & 1QZW & {$[20]$} \\
\hline & & SRP54 dimer & $\mathrm{X}$-Ray & 1QZX & {$[20]$} \\
\hline & & SRP54 with signal peptide & X-Ray & $3 \mathrm{KL} 4$ & {$[21]$} \\
\hline & & SRP19 with helix 6 and helix 8 & $\mathrm{X}$-Ray & 3KTV,3KTW & {$[22]$} \\
\hline \multirow[t]{9}{*}{ Euryarchaeota } & Archaeoglobus fulgidus & SRP19 & NMR & $1 \mathrm{KVN}, 1 \mathrm{KVV}$ & {$[23]$} \\
\hline & & SRP54M & NMR & 2JQE & {$[24]$} \\
\hline & Methanococcus jannaschii & SRP19 with SRP54 with RNA & $\mathrm{X}$-Ray & $2 \mathrm{~V} 3 \mathrm{C}$ & {$[25]$} \\
\hline & & SRP19 with helix 6 and helix 8 & X-Ray & $1 \mathrm{LNG}$ & {$[26]$} \\
\hline & & Helix 6 and helix 8 & $\mathrm{X}$-Ray & $1 \mathrm{Z} 43$ & [27] \\
\hline & & PCC & X-Ray & 1RHZ,1RH5 & {$[28]$} \\
\hline & Pyrococcus furiosus & SRP19 & $\mathrm{X}$-Ray & 3DLU,3DLV,3DM5 & [29] \\
\hline & & SRP54 & X-Ray & 3DLU,3DLV,3DM5 & [29] \\
\hline & & FtsY & X-Ray & 3E70,3DM9,3DMD & {$[30]$} \\
\hline
\end{tabular}

TABLE 2: Taxonomic distribution of archaea SRP features. Indicated are the archaea subdomains, the number of species identified in each group, and a representative species. Features are the UGUNR motif ( $\mathrm{N}$ is any nucleotide and $\mathrm{R}$ is a purine residue), helices (typically 1 to 4) in the small SRP domain (SD), the GNAR tetranucleotide loop (tetraloop) of helix 6, the GGAA tetraloop of helix 8, proteins SRP19 and SRP54 (SRP19/54), and the acidic (A) domain of the FtsY receptor (FtsY-A). "+" shows presence, "-" absence, and " \pm " indicates that this feature is present only in a subset of the group members. Sequences deviating from the top-listed motif (e.g., the GGGA in the Thermoproteales) are given. The structural alignments of the 81 identified archaea SRP RNAs and the protein alignments of SRP19, SRP54 and FtsY are provided online as listed in Supplementary Materials 2 available online at doi:10.1155/2010/485051 and are available at http://rnp.uthct.edu/rnp/SRPDB/srprna.html. Features of the SRP RNA in all three domains of life have been recently described in detail in $[1]$.

\begin{tabular}{|c|c|c|c|c|c|c|c|c|}
\hline Subdomains & Groups (n) & Prototypical Species & UGUNR & SD helices & GNAR & GGAA & SRP19/54 & FtsY-A \\
\hline \multirow[t]{3}{*}{ Crenarchaeota } & Desulfurococcales (5) & Pyrodictium occultum & - & $\begin{array}{l}\text { some } \\
\text { absent }\end{array}$ & + & + & + & - \\
\hline & Sulfolobales (12) & Sulfolobus solfataricus & \pm & + & + & + & + & + , varies \\
\hline & Thermoproteales (4) & $\begin{array}{l}\text { Thermoproteus } \\
\text { neutrophilus V24Sta }\end{array}$ & - & + & + & GGGA & + & - \\
\hline \multirow[t]{9}{*}{ Euryarchaeota } & Archaeoglobales (2) & Archaeoglobus fulgidus & + & + & + & + & + & \pm \\
\hline & Halobacteriales (10) & $\begin{array}{l}\text { Halobacterium species } \\
\text { NRC-1 }\end{array}$ & - & + & + & + & + & $\begin{array}{l} \pm \text {, some } \\
\text { long }\end{array}$ \\
\hline & Methanobacteriales (3) & $\begin{array}{l}\text { Methanobacterium } \\
\text { thermoautotrophicum }\end{array}$ & + & + & + & + & + & $\begin{array}{l}+ \text {, some } \\
\text { long }\end{array}$ \\
\hline & Methanococcales (12) & $\begin{array}{l}\text { Methanocaldococcus } \\
\text { jannaschii DSM } 2661\end{array}$ & + & + & varies & + & + & \pm \\
\hline & $\begin{array}{l}\text { Methanomicrobiales } \\
\text { (1) }\end{array}$ & $\begin{array}{l}\text { Methanoculleus } \\
\text { marisnigri JR1 }\end{array}$ & UAUAA & + & + & + & + & \pm \\
\hline & Methanosarcinales (5) & $\begin{array}{l}\text { Methanosarcina } \\
\text { acetivorans }\end{array}$ & + & + & + & + & + & \pm \\
\hline & Methanopyrales (1) & $\begin{array}{l}\text { Methanopyrus kandleri } \\
\text { AV19 }\end{array}$ & + & + & + & GAGA & + & - \\
\hline & Thermococcales (14) & $\begin{array}{l}\text { Pyrococcus horikoshii } \\
\text { OT3 }\end{array}$ & + & + & + & + & + & $\begin{array}{l} \pm \text {, some } \\
\text { long }\end{array}$ \\
\hline & Thermoplasmatales (3) & $\begin{array}{l}\text { Thermoplasma } \\
\text { acidophilum }\end{array}$ & - & + & AAAG & + & + & - \\
\hline Korarchaeota & $\begin{array}{l}\text { Candidatus } \\
\text { Korarchaeum (1) }\end{array}$ & $\begin{array}{l}\text { Candidatus Korarchaeum } \\
\text { cryptofilum OPF8 }\end{array}$ & - & + & + & + & + & - \\
\hline Thaumarchaeota & Nitrosopumilales (1) & $\begin{array}{l}\text { Nitrosopumilus } \\
\text { maritimus SCM1 }\end{array}$ & - & $\begin{array}{l}\text { some } \\
\text { absent }\end{array}$ & + & GGGA & + & - \\
\hline
\end{tabular}




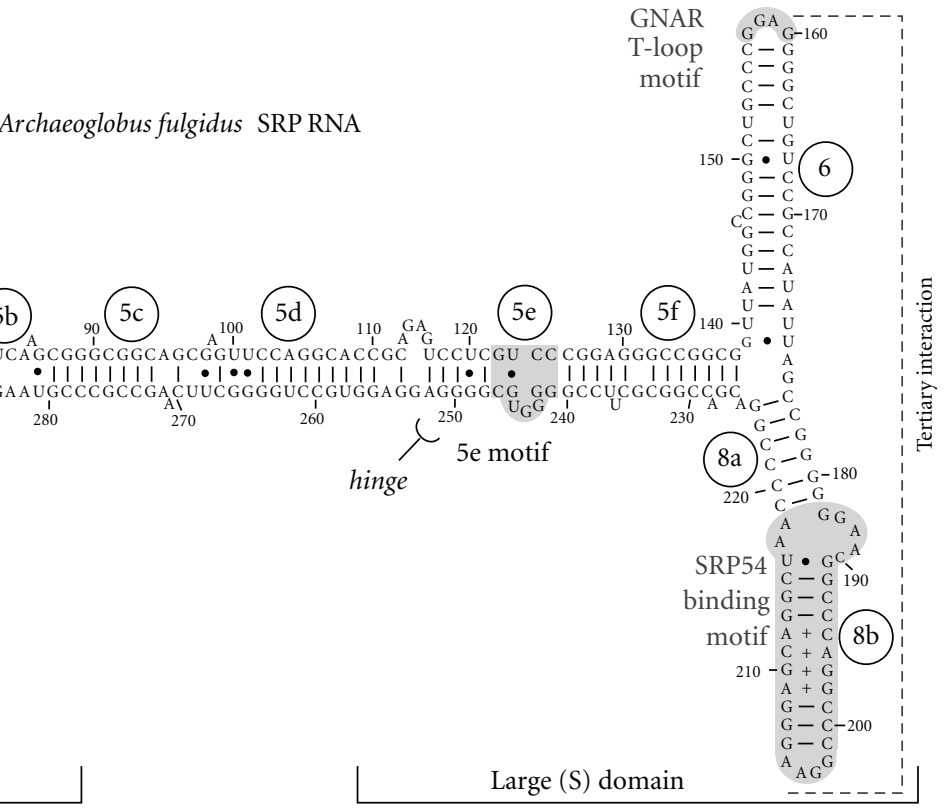

\section{Small (Alu) domain}

Consensus<smiles>[18O]=CN[Hg]</smiles><smiles>C1=C[C@H]2CC1[C@H]1C=C[C@H]2C1</smiles>

C N G G

$\mathrm{NNN}$

Archaeoglobales

G U - - C C

l l l

C G G G

UGG

\begin{abstract}
Sulfolobales
\end{abstract}
G C - - C C

l l 1

C N G G

$\mathrm{NCU}$
Thermoproteales

(a)

Eukaryota

G N - C C

I I I

C N G G

N N A

$$
\begin{aligned}
& \text { Halobacteriales } \\
& \begin{array}{c}
\text { G U - - C C } \\
\text { | I I I I G G } \\
\text { C G R }
\end{array}
\end{aligned}
$$
Methanosarcinales
G U - - Y C
$\begin{array}{lll}\mid & \mid \\ C A & R\end{array}$
Y Y R

C N G G

C C G
Protein SRP72

$$
\begin{aligned}
& \text { G U - - C C } \\
& \text { l l | } \\
& \text { A } \mathrm{R} \mathrm{G}
\end{aligned}
$$

Thermococcales

G U - - C C
$\mid \begin{gathered}\mid \\ \text { C A }\end{gathered}$ R G
N NG

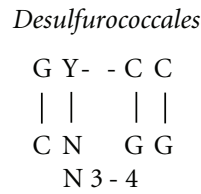

(b)

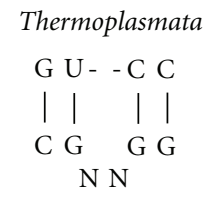

Korarchaeota

G U - - C C<smiles>C1CCCCC1</smiles>

(c)

FIgURE 2: Archaea SRP RNA features. (a) Secondary structure of Archaeoglobus fulgidus SRP RNA. (b) Consensus-matching 5e motifs sorted by their taxonomic membership. (c) Mismatching 5e motifs. Helices are numbered from 1 to 8 and helical sections are labeled with letters a to $\mathrm{f}$ [33]. For example, helix 1 is composed of residues one to seven which are base paired with residues 303 to 310 ; helix 2 consists of residues at positions ten to 13 base paired with the residues at positions 59 to 62 . The extended helix 5 contains six helical sections, $5 \mathrm{a}$ to 5 f. Helix 7 is lacking in the SRP RNAs of the archaea. The $5^{\prime}$ - and $3^{\prime}$-ends are shown, and residues are labeled in ten-residue increments. Base pairs were determined by comparative sequence analysis [32] and by considering high-resolution data (Table 1). The approximate extents of the large (S) and the small (Alu) domains are indicated. Shown in dark gray are the UGUNR motif (labeled UGU) in the small domain, the 5e motif within helical section 5e at the indicated hinge [34], the GNAR apical tetraloop of helix 6 and the SRP54 binding motif of helix 8 in the large domain. Dashed lines suggest tertiary interactions.

(A159 and A205 in Archaeoglobus fulgidus, Figure 2(a)). This long-range interaction was first seen in the crystal structures of Methanococcus jannaschii SRP RNA from the large domain (Table 1). The adenosine clamp severely constraints the arrangement of helices 6 and 8. It is highly conserved and likely exists in all archaea and eukaryotic SRP RNAs. The participating adenosine of helix 6 is presented within a GNAR tetranucleotide loop (tetraloop) in most archaea SRP RNAs, but deviates (AAAG) from the consensus in the four SRP RNA sequences of the Thermoplasmatales. The 
interacting helix 8 has a GRRA loop with GGAA being the most frequently represented tetranucleotide. GGGA is found in the Thermoproteales and Thaumarchaeota (Nitrosopumilales), and GAGA in the Methanopyrales. These helix 8 tetraloop sequences are probably useful when attempting to identify and classify the archaea SRP RNAs (Table 2).

\section{The 5e Motif: A Case for Molecular Exaptation}

The 11-nucleotide 5e element is the most recently discovered SRP RNA motif and has been helpful in the prediction of SRP RNA genes [38]. The motif consists of four base pairs interrupted by a three-nucleotide loop. Two of the base pairs are symmetrically arranged G-C pairs. The comparison of 141 eukaryal and 28 archaea sequences shows that the first residue of the eukaryotic 5e loop is a conserved adenosine (A240 in human SRP RNA) in the eukarya (Figure 2(b)) [39]. In the archaea, the corresponding nucleotide can be any residue, and only two halobacterial sequences (Haloferax volcanii, GenBank Accession AF395888, and Halomicrobium mukohataei, GenBank Accession CP001688) possess an adenosine.

Systematic site-directed mutagenesis of the 5e region showed that human SRP RNA with a single A240G change was unable to form a complex with full-length human SRP72 [39]. The 5e RNA was found to bind a 56 amino acidresidue polypeptide of human SRP72 which contained the consensus sequence PDPXRWLPXXER ( $\mathrm{X}$ is for any amino acid residue) [40]. Bioinformatic analyses identified two relatively poor consensus sequence matches in the genomes of archaea, one with a methyl coenzyme $M$ reductase of an uncultured methanogenic archaeon (GenBank Accession ABI18429), the other with a hypothetical protein of Pyrobaculum islandicum DSM 4184 (GenBank Accession ABL88435). These relationships are likely coincidental and, until proven otherwise, are consistent with the notion that a functional equivalent of the eukaryotic SRP72 is lacking in the archaea.

The conserved adenosine in the 5e motif of the eukaryal, but not the archaeal SRP RNAs suggests that the 5e element was recruited in evolution to supply a new function to the protein-rich eukaryotic SRP thereby providing a striking example for molecular exaptation, defined as the utilization of a feature for a function which differs from what it was originally developed for [41, 42]. Because human SRP72 binds strongly to the Haloferax volcanii SRP RNA [40], the structures of the 5e region of archaea and eukaryotes are apparently very similar.

The 5e RNA fragment is remarkably resistant towards ribonucleolytic attack [39] indicating that it is compactly folded and may resemble the structure of an RNA kinkturn [43]. Although 5e conforms only loosely to the Kturn consensus secondary structure, 3D molecular modeling demonstrates compatible structures (Zwieb, unpublished). This suggest that $5 \mathrm{e}$ is part of the bend or hinge which allows the elongated SRP to adjust to the curvature of the ribosome and bind simultaneously to separate ribosomal sites [34].
Such an interpretation is supported by the finding that $5 \mathrm{e}$ is present in SRP RNAs with a standard set of helices in their small SRP domain [38]. Conversely, Figure 2(c) and the data shown in Table 2 suggest that SRP RNAs deviate from the 5 e consensus when they lack the UGUNR motif or when the small SRP domain is eroded. These hinge-impaired archaea SRP RNAs may function in a mode which resembles the SRPmediated protein targeting of the majority of bacteria which lack the small SRP domain.

\section{Protein SRP19, Is It Required?}

Although protein SRP19 was thought to be absent in certain archaea genomes [3], its genes (91 sequences) have now been identified in all archaea subgroups (Table 2). SRP19 coexist with SRP RNA helix 6 as part of the large SRP domain. Mainly due to the reduced size of its loop 4, the archaea SRP19 is generally somewhat shorter than its eukaryotic homolog (Figure 3, top, gray triangle). The NMR structure of Archaeoglobus fulgidus has been solved [23], and several crystal structures of the free and RNA-bound SRP19 have been determined (Table 1) revealing a single-domain compactly folded protein.

Certain conserved amino acid residues ( $\mathrm{Y} / \mathrm{W}$ and GR in loop 1; Figure 3, top) participate in the binding to the SRP RNA through induced fit mechanisms involving both the protein and the RNA. For example, loop 3 (Figure 3, top) of Archaeoglobus fulgidus SRP19 reorders and adopts a single conformation upon binding to RNA [23]. In the Thermococcales, loop 3 is enlarged and disordered and, upon binding, rearranges to assist in the proper folding of the SRP RNA [29]. This mechanism of mutual conformational adjustment has been observed in several other protein-RNA complexes [44].

In eukaryotic cells, SRP is assembled in the nucleolus and transported to the cytosol where it associates with SRP54 $[45,46]$. Archaea SRPs contain only two proteins, SRP19 and SRP54, and assemble in the cytosol. The mammalian SRP19 is required to position SRP RNA helices 6 and 8 in a side-by-side fashion and expose the SRP54 binding site through a conformational collapse in helix 8. In contrast, archaea SRP RNA binds SRP54 even in the absence of SRP19 [47, 48]. RNase susceptibility measurements of wildtype and mutant Archaeoglobus fulgidus SRP RNAs show that the conserved adenosine of the GNAR tetraloop in helix 6, and not SRP19, is responsible for a compactly arranged large SRP domain [49]. Indeed, helices 6 and 8 are closely packed in the protein-free crystal structures of Methanococcus jannaschii and Sulfolobus solfataricus SRP RNAs $[22,27]$.

Figure 4 indicates that helix 6 and helix 8 interact with each other not only through their distal tetraloop adenosines but also via internal looped-out residues. However, the asymmetric internal loop of helix 8 engages in distinctly different ways. In the human SRP RNA, two adenosines protrude from the short strand of the asymmetric loop to form A-minor motifs with helix 6 [50]. In contrast, in the Methanococcus jannaschii RNA structures, two adenosines of 


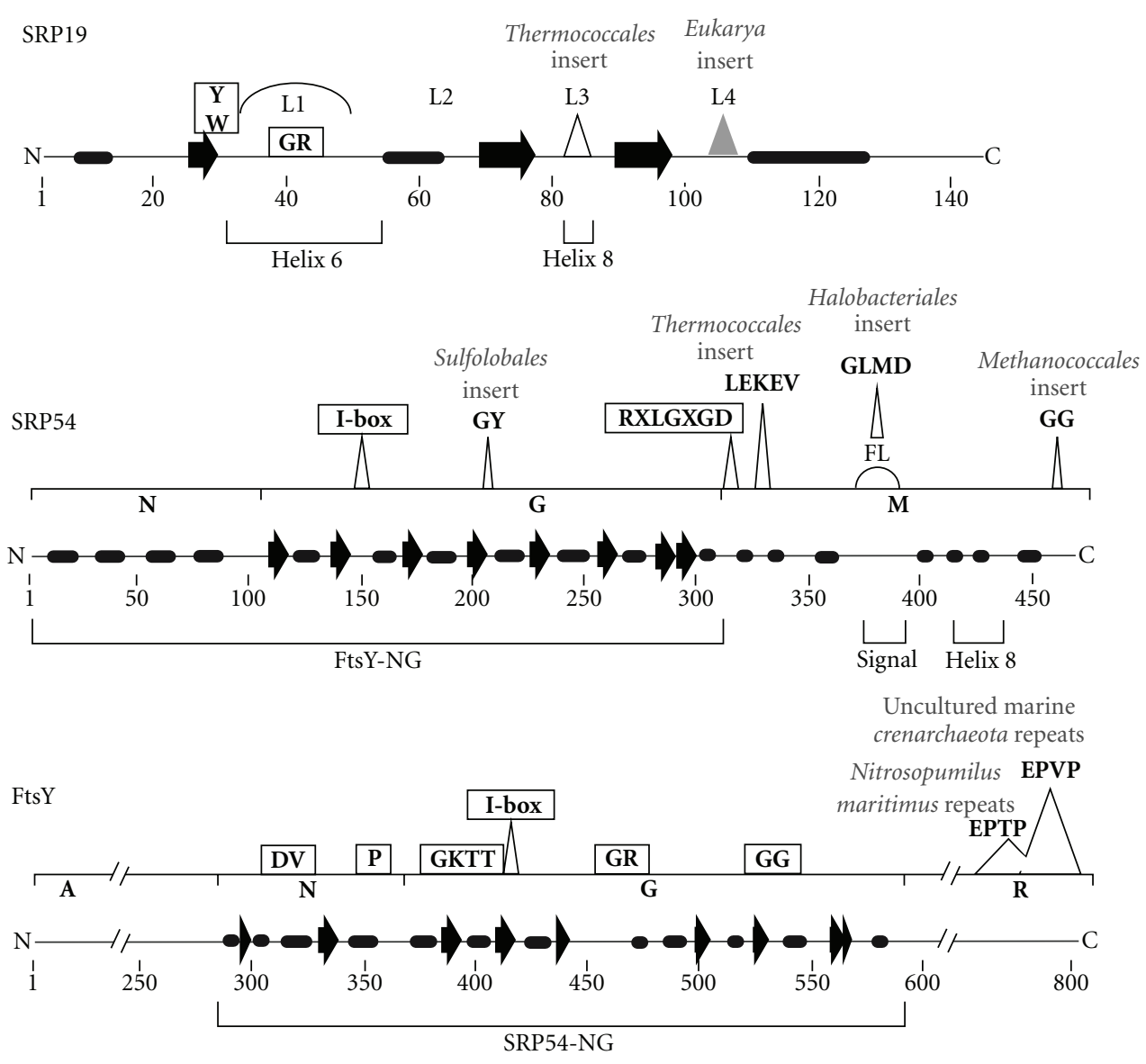

Figure 3: Features of SRP19 (top), SRP54 (center) and the FtsY SRP receptor (bottom). Indicated are the N- and C-termini. Helices are shown as cylinders, beta-sheets as arrows, and some loops are labeled with an arch and the letter L. Invariant or highly conserved residues are shown boxed, and several amino acid residue inserts which are characteristic for the indicated taxonomic groups are shown as bold letters. Numbering is according to the column positions of each protein alignment accessible as listed in Supplementary Materials 2 and at the SRP database at http://rnp.uthct.edu/rnp/SRPDB/SRPDB.html). Regions and sites which interact with other SRP components or the signal sequence are marked with brackets below each panel.

helix 6 are bulged out and interact in the minor groove of helix 8 [25].

Deletion of the yeast SRP19 homolog Sec65 was shown to be lethal to the eukaryote Yarrowia lipolytica [51]. In the archaea, structural and biochemical data as well as the deviation from the GNAR tetraloop motif observed within the Thermoplasmatales (Table 2) suggest that SRP19 is not required for SRP assembly and dispensable for protein sorting and survival. In fact, deletion of SRP19 from the Haloferax volcanii genome had no effect on protein translocation or membrane insertion. Increased levels of membrane bacterioruberin were detected in the deletion mutant and significant amounts of SRP19 mRNA were observed in nonmutated cells [52] suggesting a relatively minor possibly regulatory function for SRP19. Although the protein might participate in a more substantive way when Haloferax volcanii is challenged to survive in external environments, the data demonstrate the diminished importance of the archaea SRP19 when compared to its significant role for the survival of eukaryotic cells.

\section{SRP54}

SRP54, or its bacterial homolog Ffh, is present in all organisms, including the chloroplast SRPs which lack an SRP RNA [2]. Deletion of the Haloferax volcanii SRP54 gene results in the loss of cell viability as proof of the central role of SRP54 in archaea protein targeting $[52,53]$. Sequence and threedimensional structure (Table 1) of the protein are highly conserved. These properties are readily explained by the numerous interactions which engage SRP54 in the binding not only to the SRP RNA, but also the signal sequence and the FtsY SRP receptor. The observed exceptionally high level of conservation likely reflects the need to carry out multiple binding reactions in a coordinated dynamically GTP-regulated way to ensure proper and efficient delivery of a wide variety of signal sequence-tagged proteins into the PCC.

The functions of SRP54 are brought about by three domains. The N-terminal $(\mathrm{N})$ domain is composed of a bundle of four alpha helices, the GTPase $(\mathrm{G})$ domain 


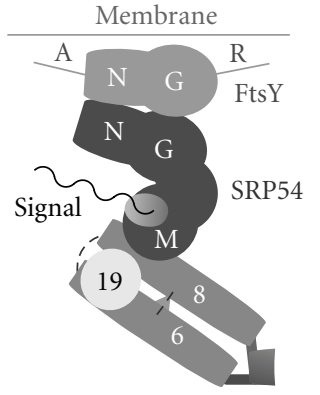

FIGURE 4: Interactions between the components of archaea SRPmediated protein targeting. Schematic drawing of the coaxiallyarranged SRP RNA helices 6 and 8 bound together by SRP19 and two tertiary interactions (dashed lines). The M-domain of the SRP54 protein (dark gray) binds to SRP RNA helix 8 as well as the signal sequence (black). The NG-domains of SRP54 and the FtsY SRP receptor are arranged quasisymmetrically and poised to separate upon the hydrolysis of two G-domain-bound GTP molecules. The N-terminal region labeled $\mathrm{A}$ (for acidic) and the $\mathrm{C}$ terminal repeat region ( $\mathrm{R}$ ) of FtsY are variable or may be absent (see Table 2).

contains a unique insertion (I-box) which serves as a guanine nucleotide-exchange factors (GEFs) and stabilizes the nucleotide free protein $[54,55]$, and the methioninerich $(\mathrm{M})$ domain binds to the SRP RNA and the signal sequence (Figure 3, center). The predominantly alpha helical $\mathrm{M}$ domain contains an extended segment (the so-called fingerloop) which delineates or is folded into a groove which accepts signal sequences $[20,21,56]$. This wide and short hydrophobic groove was observed also in the crystal structure of the RNA-bound Escherichia coli Ffh [57]. The NMR structure of the Archaeoglobus fulgidus SRP54 M domain [24] is similar to these crystal structures and disfavors another proposed mode whereby the signal sequence binds within a long and narrow groove of SRP54M $[28,50]$. The conformations of the fingerloop in solution suggest that it adaptively binds and stabilizes the signal sequences. Binding is weak [24] and likely reversible in order to permit signal sequence release upon the binding of SRP54 to the SRP receptor. The molecular details of the contacts made by a signal peptide with the Sulfolobus solfataricus SRP54 have been revealed recently and suggest that portions of the fingerloop may adopt an alpha helical conformation [21].

Adding to the intricacy of signal sequence recognition, the $\mathrm{M}$ domain and the NG region of SRP54 are joined together via a flexible linker. This region has the consensus sequence RXLGXGD and allows the RNA-bound SRP54 to undergo substantial structural rearrangements upon binding to a signal sequence $[20,22]$. Consistent with this assertion, site-directed mutagenesis experiments of mammalian SRP [58] and a recent crosslinking study of the Escherichia coli SRP [59] demonstrate the involvement of the signal sequence not only with the $\mathrm{M}$ domain, but also the NG region. No evidence for the binding of $\mathrm{NG}$ to signal sequences has been provided in the archaea. However, the exceptional conservation of SRP54 throughout all domains of life suggests that archaea employ a similar if not identical signal recognition mechanism. The NG region can be in close proximity to SRP RNA helix 8 and, in archaea, appears to engage also helix 6 [25].

The alignment of 103 archaea SRP54 sequences reveals several group-specific amino acid residue insertions, for example a GY in the G domain of Sulfolobales which might modulate the GTPase activity. Into the $\mathrm{M}$ domain, Thermococcales insert the sequence LEKEV, Halobacteriales GLMD, and Methanococcales GG (Figure 3). These amino acid residues have the potential to contribute to the binding of the protein to the SRP RNA, to signal peptide recognition or other yet to be specified enhanced functions. Regardless of their potential significance, these short peptide sequences are useful for assigning SRP54 sequences to their proper taxonomic group.

\section{FtsY: The SRP Receptor}

The SRP receptor (SR) of the eukarya is composed of the peripheral membrane $\mathrm{SR} \alpha$ and the integral membrane $\mathrm{SR} \beta$ proteins. Bacteria and archaea possess only FtsY, a homolog of SR $\alpha$ [60]. Sequence comparisons of FtsY with SRP54 suggest a gene duplication event [61] and support the classification into the three domains of life as well as the close rooting of archaea and eukarya [62]. Archaea FtsY shares its conserved NG region with NG of SRP54, including the I-box, but differs from SRP54 with respect to several short amino acid stretches as revealed by the alignment of 95 archaea FtsY sequences (Figure 3, Supplementary Material 1). The NG regions are symmetrically arranged in three dimensions to constitute the structural and functional core of signal sequence release and nascent polypeptide delivery into the cell membrane (Figure 4) by mutually catalyzing the hydrolysis of GTP [63-65].

As has been observed within the bacterial genomes $[66,67]$ several archaea FtsY sequences consist only of the NG domain and lack an N-terminal acidic (A) domain. Diversity with respect to the A domain is observed even within a single archaea subgroup (Table 2). Full-length Haloferax volcanii FtsY as well as polypeptides lacking the A domain were shown to bind to inverted membrane vesicles indicating that the A domain is dispensable for attaching FtsY to the membrane. Instead, the A domain may play a role in recruiting SRP to the haloarchaeal membrane $[68,69]$. Assuming a pool of free FtsY in the cytosol $[70,71]$ (Figure 1) these findings are particularly relevant. On the other hand, fluorescence microscopy showed that almost all of the Escherichia coli FtsY associates in vivo with the inner membrane, and any soluble FtsY is unlikely to contribute to protein targeting [72]. Although archaea FtsY might interact with the membrane in similar manners as has been observed in bacteria and chloroplast [2, 73-76], the molecular details of the binding could be quite different given the differences in membrane lipid composition. FtsY might also interact directly with a cytosolically exposed portion of the PCC $[77,78]$. In either case, one would 
expect functional synchronicity between GTP hydrolysis and delivery of protein into the PCC [79].

In the FtsY sequences of the uncultured marine Crenarchaeota we discovered a C-terminal proline-rich extension, named $\mathrm{R}$ for its motif repetitions (see Figures 3 and 4). Up to 12 EPVP repeats (accession numbers ABZ10052, ABZ08863, ABZ09152, ABZ09615) and five EPVV repeats (ABZ098531) were present in the R region. Similar multiple repeats with the sequence EPTP were seen also in the FtsY of the Thaumarchaeotum Nitrosopumilus maritimus SCM1. Details of the R-regions can be inspected in an updated archaeal FtsY alignment provided at the SRPDB [37]. As with much of our limited understanding of the role of FtsY in the archaea, it remains to be determined if these repeats are expressed and have a function in protein export.

\section{Archaea SRP Function and Evolution}

During the past years, several interesting puzzle pieces with respect to SRP-mediated protein translocation and membrane insertion in the archaea have been assembled. The SRPs of the Crenarchaeotum Acidianus ambivalens and the Euryarchaeota Archaeoglobus fulgidus, Pyrococcus furiosus and Haloferax volcanii have been reconstituted [47, 48, 8082], and the ability of an archaea SRP54 to participate in signal sequence recognition has been demonstrated [81]. Nevertheless, the role of SRP within the archaeal cell is still poorly understood. Examples of both protein synthesislinked (cotranslational) and posttranslational translocation have been provided [83-87], but to what degree these findings are representative remains to be investigated further [16].

The proposal that signal sequences might interact with the SRP RNA has fed the imagination that the primitive SRP was composed only of RNA [57, 88]. However, because of the proteinaceous nature of the signal, a scenario in which SRP RNA coemerged with evolutionary precursors of $\mathrm{SRP} 54 / \mathrm{Ffh} / \mathrm{FtsY}$ appears to be more plausible. Furthermore, the recent structure of the signal peptide-bound Sulfolobus solfataricus SRP54 (Ffh) shows that the signal peptide is too far removed from the SRP RNA to make direct contact [21].

If the small (Alu) SRP domain was a feature of the primitive SRP which subsequently was lost in evolutionary time; the majority of the bacteria is more difficult to discern. As another possibility archaea and certain bacteria may have been faced independently with the need to enlarge a small primitive SRP, maybe to slow down translation rates and provide more time for ensuring the delivery of proteins to the membrane as has been observed in eukarya [89].

\section{Future Directions}

With respect to the RNA-rich archaea SRP it would be desirable to better understand the structure and function of the protein-free small SRP domain. For example, what, in molecular detail, allows the small domain to fold back onto helix 5 in order to approximate the shape and dimensions of the eukaryal SRP [36]? What is the functional significance of the conserved 5e motif and its relationship to a flexible hinge or a bend in the elongated SRP? It will also be important to further elucidate the role of the archaea FtsY, its role in the cytosol as well as the molecular features which promote its association with archaea membranes. As in the past, the studies of the archaea SRP are expected to contribute in many ways to our grasp of SRP-mediated protein targeting in all organism.

\section{Methods}

Sets of representative sequences were used as input to Perl scripts written to identify sequence homologs in the NCBI databases [90]. RNA sequences were aligned semiautomatically with SARSE [91]; protein sequences were aligned using MUSCLE [92] followed by manual adjustments in Jalview [93]. The alignments are available through the links listed in Supplementary Material 2. In addition, the SRP database provides tables of alphabetically and phylogenetically sorted sequences at http://rnp.uthct.edu/rnp/SRPDB/SRPDB.html.

\section{Acknowledgments}

The authors are grateful to the American Heart Association, South Central Affiliate, for support (no. 09GRNT2080038). S.Bhuiyan is supported by a UNIMET grant from the National Aeronautics and Space Administration and the United Negro College Fund Special Programs Corporation NASA Science and Technology Institute for Minority Institution Project NNA06CB14H.

\section{References}

[1] M. A. Rosenblad, N. Larsen, T. Samuelsson, and C. Zwieb, "Kinship in the SRP RNA family," RNA Biology, vol. 6, no. 5, pp. 508-516, 2009.

[2] K. F. Stengel, I. Holdermann, P. Cain, C. Robinson, K. Wild, and I. Sinning, "Structural basis for specific substrate recognition by the chloroplast signal recognition particle protein cpSRP43," Science, vol. 321, no. 5886, pp. 253-256, 2008.

[3] C. Zwieb and J. Eichler, "Getting on target: the archaeal signal recognition particle," Archaea, vol. 1, no. 1, pp. 27-34, 2002.

[4] R. G. Moll, "The archaeal signal recognition particle: steps toward membrane binding," Journal of Bioenergetics and Biomembranes, vol. 36, no. 1, pp. 47-53, 2004.

[5] H. G. Koch, M. Moser, and M. Müller, "Signal recognition particle-dependent protein targeting, universal to all kingdoms of life," Reviews of Physiology, Biochemistry and Pharmacology, vol. 146, pp. 55-94, 2003.

[6] K. Nagai, C. Oubridge, A. Kuglstatter, E. Menichelli, C. Isel, and L. Jovine, "Structure, function and evolution of the signal recognition particle," EMBO Journal, vol. 22, no. 14, pp. 34793485, 2003.

[7] J. A. Doudna and R. T. Batey, "Structural insights into the signal recognition particle," Annual Review of Biochemistry, vol. 73, pp. 539-557, 2004.

[8] M. Halic and R. Beckmann, "The signal recognition particle and its interactions during protein targeting," Current Opinion in Structural Biology, vol. 15, no. 1, pp. 116-125, 2005. 
[9] S.-O. Shan and P. Walter, "Co-translational protein targeting by the signal recognition particle," FEBS Letters, vol. 579, no. 4, pp. 921-926, 2005.

[10] G. D. Sprott, "Structures of archaebacterial membrane lipids," Journal of Bioenergetics and Biomembranes, vol. 24, no. 6, pp. 555-566, 1992.

[11] J. L. C. M. Van de Vossenberg, A. J. M. Driessen, and W. N. Konings, "The essence of being extremophilic: the role of the unique archaeal membrane lipids," Extremophiles, vol. 2, no. 3, pp. 163-170, 1998.

[12] G. von Heijne, “The signal peptide," Journal of Membrane Biology, vol. 115, no. 3, pp. 195-201, 1990.

[13] M. Pohlschröder, M. I. Giménez, and K. F. Jarrell, "Protein transport in Archaea: Sec and twin arginine translocation pathways," Current Opinion in Microbiology, vol. 8, no. 6, pp. 713-719, 2005.

[14] S. Y. M. Ng, B. Chaban, D. J. VanDyke, and K. F. Jarrell, "Archaeal signal peptidases," Microbiology, vol. 153, no. 2, pp. 305-314, 2007.

[15] S.-V. Albers, Z. Szabó, and A. J. M. Driessen, "Protein secretion in the Archaea: multiple paths towards a unique cell surface," Nature Reviews Microbiology, vol. 4, no. 7, pp. 537-547, 2006.

[16] D. Calo and J. Eichler, "Crossing the membrane in Archaea, the third domain of life," Biochimica et Biophysica Acta. In press.

[17] J. Yuan, J. C. Zweers, J. M. van Dijl, and R. E. Dalbey, "Protein transport across and into cell membranes in bacteria and archaea," Cellular and Molecular Life Sciences, vol. 67, no. 2, pp. 179-199, 2010.

[18] A. Kouranov, L. Xie, J. de la Cruz et al., "The RCSB PDB information portal for structural genomics," Nucleic Acids Research, vol. 34, pp. D302-305, 2006.

[19] G. Montoya, K. te Kaat, R. Moll, G. Schäfer, and I. Sinning, "The crystal structure of the conserved GTPase of SRP54 from the archaeon Acidianus ambivalens and its comparison with related structures suggests a model for the SRP-SRP receptor complex," Structure, vol. 8, no. 5, pp. 515-525, 2000.

[20] K. R. Rosendal, K. Wild, G. Montoya, and I. Sinning, "Crystal structure of the complete core of archaeal signal recognition particle and implications for interdomain communication," Proceedings of the National Academy of Sciences of the United States of America, vol. 100, no. 25, pp. 14701-14706, 2003.

[21] C. Y. Janda, J. Li, C. Oubridge, H. Hernández, C. V. Robinson, and K. Nagai, "Recognition of a signal peptide by the signal recognition particle," Nature, vol. 465, no. 7297, pp. 507-510, 2010.

[22] K. Wild, G. Bange, G. Bozkurt, B. Segnitz, A. Hendricks, and I. Sinning, "Structural insights into the assembly of the human and archaeal signal recognition particles," Acta Crystallographica Section D, vol. 66, no. 3, pp. 295-303, 2010.

[23] O. N. Pakhomova, S. Deep, Q. Huang, C. Zwieb, and A. P. Hinck, "Solution structure of protein SRP19 of Archaeoglobus fulgidus signal recognition particle," Journal of Molecular Biology, vol. 317, no. 1, pp. 145-158, 2002.

[24] U. Ilangovan, S. H. Bhuiyan, C. S. Hinck et al., "A. fulgidus SRP54 M-domain," Journal of Biomolecular NMR, vol. 41, no. 4, pp. 241-248, 2008.

[25] T. Hainz, S. Huang, and A. E. Sauer-Eriksson, "Interaction of signal-recognition particle 54 GTPase domain and signalrecognition particle RNA in the free signal-recognition particle," Proceedings of the National Academy of Sciences of the United States of America, vol. 104, no. 38, pp. 14911-14916, 2007.
[26] T. Hainzl, S. Huang, and A. E. Sauer-Eriksson, "Structure of the SRP19-RNA complex and implications for signal recognition particle assembly," Nature, vol. 417, no. 6890, pp. 767-771, 2002.

[27] T. Hainzl, S. Huang, and A. E. Sauer-Eriksson, "Structural insights into SRP RNA: an induced fit mechanism for SRP assembly," RNA, vol. 11, no. 7, pp. 1043-1050, 2005.

[28] W. M. Clemons Jr., K. Gowda, S. D. Black, C. Zwieb, and V. Ramakrishnan, "Crystal structure of the conserved subdomain of human protein SRP54M at $2.1 \AA$ resolution: evidence for the mechanism of signal peptide binding," Journal of Molecular Biology, vol. 292, no. 3, pp. 697-705, 1999.

[29] P. F. Egea, J. Napetschnig, P. Walter, and R. M. Stroud, "Structures of SRP54 and SRP19, the two proteins that organize the ribonucleic core of the signal recognition particle from Pyrococcus furiosus," PLoS One, vol. 3, no. 10, Article ID e3528, 2008.

[30] P. F. Egea, H. Tsuruta, G. P. de Leon, J. Napetschnig, P. Walter, and R. M. Stroud, "Structures of the signal recognition particle receptor from the Archaeon Pyrococcus furiosus: Implications for the targeting step at the membrane," PLoS One, vol. 3, no. 11, Article ID e3619, 2008.

[31] J. M. Kollman and R. F. Doolittle, "Determining the relative rates of change for prokaryotic and eukaryotic proteins with anciently duplicated paralogs," Journal of Molecular Evolution, vol. 51, no. 2, pp. 173-181, 2000.

[32] N. Larsen and C. Zwieb, "SRP-RNA sequence alignment and secondary structure," Nucleic Acids Research, vol. 19, no. 2, pp. 209-215, 1991.

[33] C. Zwieb, R. W. Van Nues, M. A. Rosenblad, J. D. Brown, and T. Samuelsson, "A nomenclature for all signal recognition particle RNAs," RNA, vol. 11, no. 1, pp. 7-13, 2005.

[34] M. Halic, T. Becker, M. R. Pool et al., "Structure of the signal recognition particle interacting with the elongation-arrested ribosome," Nature, vol. 427, no. 6977, pp. 808-814, 2004.

[35] D. A. Benson, I. Karsch-Mizrachi, D. J. Lipman, J. Ostell, and E. W. Sayers, "GenBank," Nucleic Acids Research, vol. 37, database issue, pp. D26-D31, 2009.

[36] O. Weichenrieder, K. Wild, K. Strub, and S. Cusack, "Structure and assembly of the Alu domain of the mammalian signal recognition particle," Nature, vol. 408, no. 6809, pp. 167-173, 2000.

[37] E. S. Andersen, M. A. Rosenblad, N. Larsen et al., "The tmRDB and SRPDB resources," Nucleic acids research., vol. 34, pp. D163-168, 2006.

[38] M. Regalia, M. A. Rosenblad, and T. Samuelsson, "Prediction of signal recognition particle RNA genes," Nucleic Acids Research, vol. 30, no. 15, pp. 3368-3377, 2002.

[39] E. Iakhiaeva, J. Wower, I. K. Wower, and C. Zwieb, "The 5e motif of eukaryotic signal recognition particle RNA contains a conserved adenosine for the binding of SRP72," RNA, vol. 14, no. 6, pp. 1143-1153, 2008.

[40] E. Iakhiaeva, J. Yin, and C. Zwieb, "Identification of an RNA-binding domain in human SRP72," Journal of Molecular Biology, vol. 345, no. 4, pp. 659-666, 2005.

[41] C. Darwin, "Modes of transition," in The Origin of Species, chapter 6, John Murray, London, UK, 1859.

[42] S. J. Gould and E. S. Vrba, "Exaptation-a missing term in the science of form," Paleobiology, vol. 8, no. 1, pp. 4-15, 1982.

[43] S. Nolivos, A. J. Carpousis, and B. Clouet-d'Orval, “The Kloop, a general feature of the Pyrococcus C/D guide RNAs, is an RNA structural motif related to the K-turn," Nucleic Acids Research, vol. 33, no. 20, pp. 6507-6514, 2005. 
[44] R. Stefl, L. Skrisovska, and F. H.-T. Allain, "RNA sequenceand shape-dependent recognition by proteins in the ribonucleoprotein particle," EMBO Reports, vol. 6, no. 1, pp. 33-38, 2005.

[45] J. C. Politz, S. Yarovoi, S. M. Kilroy, K. Gowda, C. Zwieb, and T. Pederson, "Signal recognition particle components in the nucleolus," Proceedings of the National Academy of Sciences of the United States of America, vol. 97, no. 1, pp. 55-60, 2000.

[46] T. S. Maity and K. M. Weeks, "A threefold RNA-protein interface in the signal recognition particle gates native complex assembly," Journal of Molecular Biology, vol. 369, no. 2, pp. 512-524, 2007.

[47] H. Maeshima, E. Okuno, T. Aimi, T. Morinaga, and T. Itoh, "An archaeal protein homologous to mammalian SRP54 and bacterial Ffh recognizes a highly conserved region of SRP RNA," FEBS Letters, vol. 507, no. 3, pp. 336-340, 2001.

[48] I. Tozik, Q. Huang, C. Zwieb, and J. Eichler, "Reconstitution of the signal recognition particle of the halophilic archaeon Haloferax volcanii," Nucleic Acids Research, vol. 30, no. 19, pp. 4166-4175, 2002.

[49] J. Yin, Q. Huang, O. N. Pakhomova, A. P. Hinck, and C. Zwieb, "The conserved adenosine in helix 6 of Archaeoglobus fulgidus signal recognition particle RNA initiates SRP assembly," Archaea, vol. 1, no. 4, pp. 269-275, 2004.

[50] A. Kuglstatter, C. Oubridge, and K. Nagai, "Induced structural changes of 7SL RNA during the assembly of human signal recognition particle," Nature Structural Biology, vol. 9, no. 10, pp. 740-744, 2002.

[51] M. Sánchez, J.-M. Beckerich, C. Gaillardin, and A. Domínguez, "Isolation and cloning of the Yarrowia lipolytica SEC65 gene, a component of the yeast signal recognition particle displaying homology with the human SRP19 gene," Gene, vol. 203, no. 1, pp. 75-84, 1997.

[52] S. Yurist, I. Dahan, and J. Eichler, "SRP19 is a dispensable component of the signal recognition particle in Archaea," Journal of Bacteriology, vol. 189, no. 1, pp. 276-279, 2007.

[53] R. W. Rose and M. Pohlschröder, "In vivo analysis of an essential archaeal signal recognition particle in its native host," Journal of Bacteriology, vol. 184, no. 12, pp. 3260-3267, 2002.

[54] C. Moser, O. Mol, R. S. Goody, and I. Sinning, "The signal recognition particle receptor of Escherichia coli (FtsY) has a nucleotide exchange factor built into the GTPase domain," Proceedings of the National Academy of Sciences of the United States of America, vol. 94, no. 21, pp. 11339-11344, 1997.

[55] P. J. Rapiejko and R. Gilmore, "Empty site forms of the SRP54 and SR $\alpha$ GTPase mediate targeting of ribosome-nascent chain complexes to the endoplasmic reticulum," Cell, vol. 89, no. 5, pp. 703-713, 1997.

[56] R. J. Keenan, D. M. Freymann, P. Walter, and R. M. Stroud, "Crystal structure of the signal sequence binding subunit of the signal recognition particle," Cell, vol. 94, no. 2, pp. 181191, 1998.

[57] R. T. Batey, R. P. Rambo, L. Lucast, B. Rha, and J. A. Doudna, "Crystal structure of the ribonucleoprotein core of the signal recognition particle," Science, vol. 287, no. 5456, pp. 1232$1239,2000$.

[58] J. A. Newitt and H. D. Bernstein, "The N-domain of the signal recognition particle $54-\mathrm{kDa}$ subunit promotes efficient signal sequence binding," European Journal of Biochemistry, vol. 245, no. 3, pp. 720-729, 1997.

[59] E. M. Clérico, A. Szymanska, and L. M. Gierasch, "Exploring the interactions between signal sequences and E. coli SRP by two distinct and complementary crosslinking methods," Biopolymers, vol. 92, no. 3, pp. 201-211, 2009.
[60] J. D. Miller, H. D. Bernstein, and P. Walter, "Interaction of E. coli Ffh/4.5S ribonucleoprotein and FtsY mimics that of mammalian signal recognition particle and its receptor," Nature, vol. 367, no. 6464, pp. 657-659, 1994.

[61] S. Althoff, D. Selinger, and J. A. Wise, "Molecular evolution of SRP cycle components: functional implications," Nucleic Acids Research, vol. 22, no. 11, pp. 1933-1947, 1994.

[62] S. Gribaldo and P. Cammarano, "The root of the universal tree of life inferred from anciently duplicated genes encoding components of the protein-targeting machinery," Journal of Molecular Evolution, vol. 47, no. 5, pp. 508-516, 1998.

[63] P. F. Egea, S.-O. Shan, J. Napetschnig, D. F. Savage, P. Walter, and R. M. Stroud, "Substrate twinning activates the signal recognition particle and its receptor," Nature, vol. 427, no. 6971, pp. 215-221, 2004.

[64] A. Zelazny, A. Seluanov, A. Cooper, and E. Bibi, "The NG domain of the prokaryotic signal recognition particle receptor, FtsY, is fully functional when fused to an unrelated integral membrane polypeptide," Proceedings of the National Academy of Sciences of the United States of America, vol. 94, no. 12, pp. 6025-6029, 1997.

[65] E. Bibi, A. A. Herskovits, E. S. Bochkareva, and A. Zelazny, "Putative integral membrane SRP receptors," Trends in Biochemical Sciences, vol. 26, no. 1, pp. 15-16, 2001.

[66] S. A. Ladefoged and G. Christiansen, "A GTP-binding protein of Mycoplasma hominis: a small sized homolog to the signal recognition particle receptor FtsY," Gene, vol. 201, no. 1-2, pp. 37-44, 1997.

[67] I. V. Shepotinovskaya and D. M. Freymann, "Conformational change of the $\mathrm{N}$-domain on formation of the complex between the GTPase domains of Thermus aquaticus Ffh and FtsY," Biochimica et Biophysica Acta, vol. 1597, no. 1, pp. 107-114, 2002.

[68] T. Lichi, G. Ring, and J. Eichler, "Membrane binding of SRP pathway components in the halophilic archaea Haloferax volcanii," European Journal of Biochemistry, vol. 271, no. 7, pp. 1382-1390, 2004.

[69] A. Haddad, R. W. Rose, and M. Pohlschröder, "The Haloferax volcanii FtsY homolog is critical for haloarchaeal growth but does not require the A domain," Journal of Bacteriology, vol. 187, no. 12, pp. 4015-4022, 2005.

[70] J. Luirink, C. M. Ten Hagen-Jongman, C. C. van der Weijden et al., "An alternative protein targeting pathway in Escherichia coli: Studies on the role of FtsY," EMBO Journal, vol. 13, no. 10, pp. 2289-2296, 1994.

[71] H.-J. Dong, J.-Y. Jiang, and Y.-Q. Li, "The distinct anchoring mechanism of FtsY from different microbes," Current Microbiology, vol. 59, no. 3, pp. 336-340, 2009.

[72] M. Mircheva, D. Boy, B. Weiche, F. Hucke, P. Graumann, and H.-G. Koch, "Predominant membrane localization is an essential feature of the bacterial signal recognition particle receptor," BMC Biology, vol. 7, article no. 76, 2009.

[73] E. de Leeuw, D. Poland, O. Mol et al., "Membrane association of FtsY, the E. coli SRP receptor," FEBS Letters, vol. 416, no. 3, pp. 225-229, 1997.

[74] E. de Leeuw, K. te Kaat, C. Moser et al., "Anionic phospholipids are involved in membrane association of FtsY and stimulate its GTPase activity," EMBO Journal, vol. 19, no. 4, pp. 531-541, 2000.

[75] R. Parlitz, A. Eitan, G. Stjepanovic et al., "Escherichia coli signal recognition particle receptor FtsY contains an essential and autonomous membrane-binding amphipathic helix," Journal of Biological Chemistry, vol. 282, no. 44, pp. 3217632184, 2007. 
[76] N. J. Marty, D. Rajalingam, A. D. Kight et al., "The membranebinding motif of the chloroplast signal recognition particle receptor (cpFtsY) regulates GTPase activity," Journal of Biological Chemistry, vol. 284, no. 22, pp. 14891-14903, 2009.

[77] S. Angelini, S. Deitermann, and H.-G. Koch, "FtsY, the bacterial signal-recognition particle receptor, interacts functionally and physically with the SecYEG translocon," EMBO Reports, vol. 6, no. 5, pp. 476-481, 2005.

[78] B. Weiche, J. Bürk, S. Angelini, E. Schiltz, J. O. Thumfart, and H.-G. Koch, "A cleavable N-terminal membrane anchor is involved in membrane binding of the Escherichia coli SRP receptor," Journal of Molecular Biology, vol. 377, no. 3, pp. 761773, 2008.

[79] L. Bahari, R. Parlitz, A. Eitan et al., "Membrane targeting of ribosomes and their release require distinct and separable functions of FtsY," Journal of Biological Chemistry, vol. 282, no. 44, pp. 32168-32175, 2007.

[80] R. Moll, S. Schmidtke, and G. Schäfer, "Domain structure, GTP-hydrolyzing activity and 7S RNA binding of Acidianus ambivalens Ffh-homologous protein suggest an SRP-like complex in archaea," European Journal of Biochemistry, vol. 259, no. 1-2, pp. 441-448, 1999.

[81] S. H. Bhuiyan, K. Gowda, H. Hotokezaka, and C. Zwieb, "Assembly of archaeal signal recognition particle from recombinant components," Nucleic Acids Research, vol. 28, no. 6, pp. 1365-1373, 2000.

[82] J. L. Diener and C. Wilson, "Role of SRP19 in assembly of the Archaeoglobus fulgidus signal recognition particle," Biochemistry, vol. 39, no. 42, pp. 12862-12874, 2000.

[83] R. Gropp, F. Gropp, and M. C. Betlach, "Association of the halobacterial 7S RNA to the polysome correlates with expression of the membrane protein bacterioopsin," Proceedings of the National Academy of Sciences of the United States of America, vol. 89, no. 4, pp. 1204-1208, 1992.

[84] H. Dale and M. P. Krebs, "Membrane insertion kinetics of a protein domain in vivo. The bacterioopsin $\mathrm{N}$ terminus inserts co-translationally," Journal of Biological Chemistry, vol. 274, no. 32, pp. 22693-22698, 1999.

[85] H. Dale, C. M. Angevine, and M. P. Krebs, "Ordered membrane insertion of an archaeal opsin in vivo," Proceedings of the National Academy of Sciences of the United States of America, vol. 97, no. 14, pp. 7847-7852, 2000.

[86] G. Ring and J. Eichler, "Extreme secretion: protein translocation across the archaleal plasma membrane," Journal of Bioenergetics and Biomembranes, vol. 36, no. 1, pp. 35-45, 2004.

[87] R. Ortenberg and M. Mevarech, "Evidence for posttranslational membrane insertion of the integral membrane protein bacterioopsin expressed in the heterologous halophilic archaeon Haloferax volcanii," Journal of Biological Chemistry, vol. 275, no. 30, pp. 22839-22846, 2000.

[88] K. Wild, M. Halic, I. Sinning, and R. Beckmann, "SRP meets the ribosome," Nature Structural and Molecular Biology, vol. 11, no. 11, pp. 1049-1053, 2004.

[89] A. K. K. Lakkaraju, C. Mary, A. Scherrer, A. E. Johnson, and K. Strub, "SRP keeps polypeptides translocation-competent by slowing translation to match limiting ER-targeting sites," Cell, vol. 133, no. 3, pp. 440-451, 2008.

[90] S. McGinnis and T. L. Madden, "BLAST: at the core of a powerful and diverse set of sequence analysis tools," Nucleic Acids Research, vol. 32, pp. W20-W25, 2004.

[91] E. S. Andersen, A. Lind-Thomsen, B. Knudsen et al., "Semiautomated improvement of RNA alignments," RNA, vol. 13, no. 11, pp. 1850-1859, 2007.
[92] R. C. Edgar, "MUSCLE: multiple sequence alignment with high accuracy and high throughput," Nucleic Acids Research, vol. 32, no. 5, pp. 1792-1797, 2004.

[93] M. Clamp, J. Cuff, S. M. Searle, and G. J. Barton, “The Jalview Java alignment editor," Bioinformatics, vol. 20, no. 3, pp. 426427, 2004. 

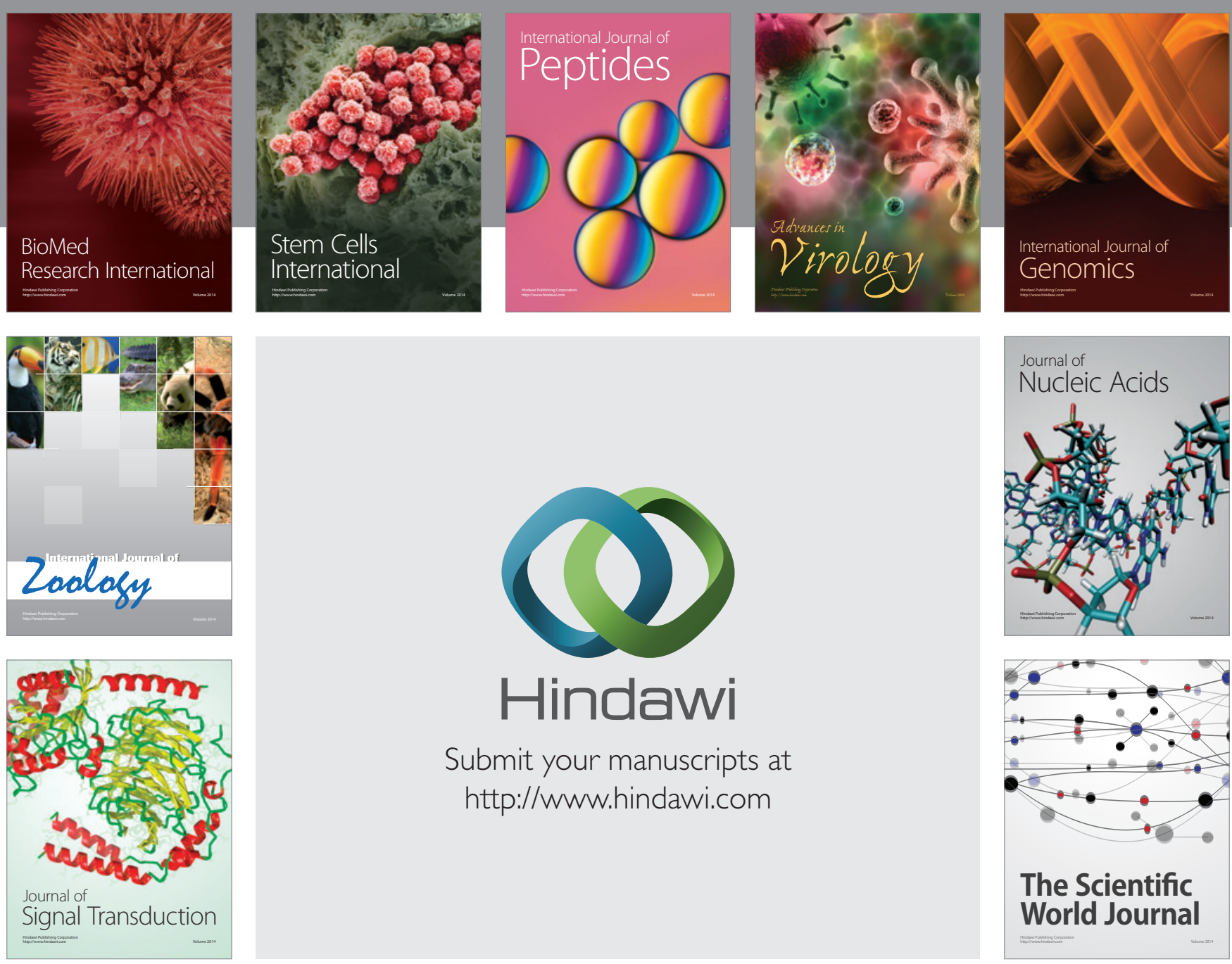

Submit your manuscripts at

http://www.hindawi.com
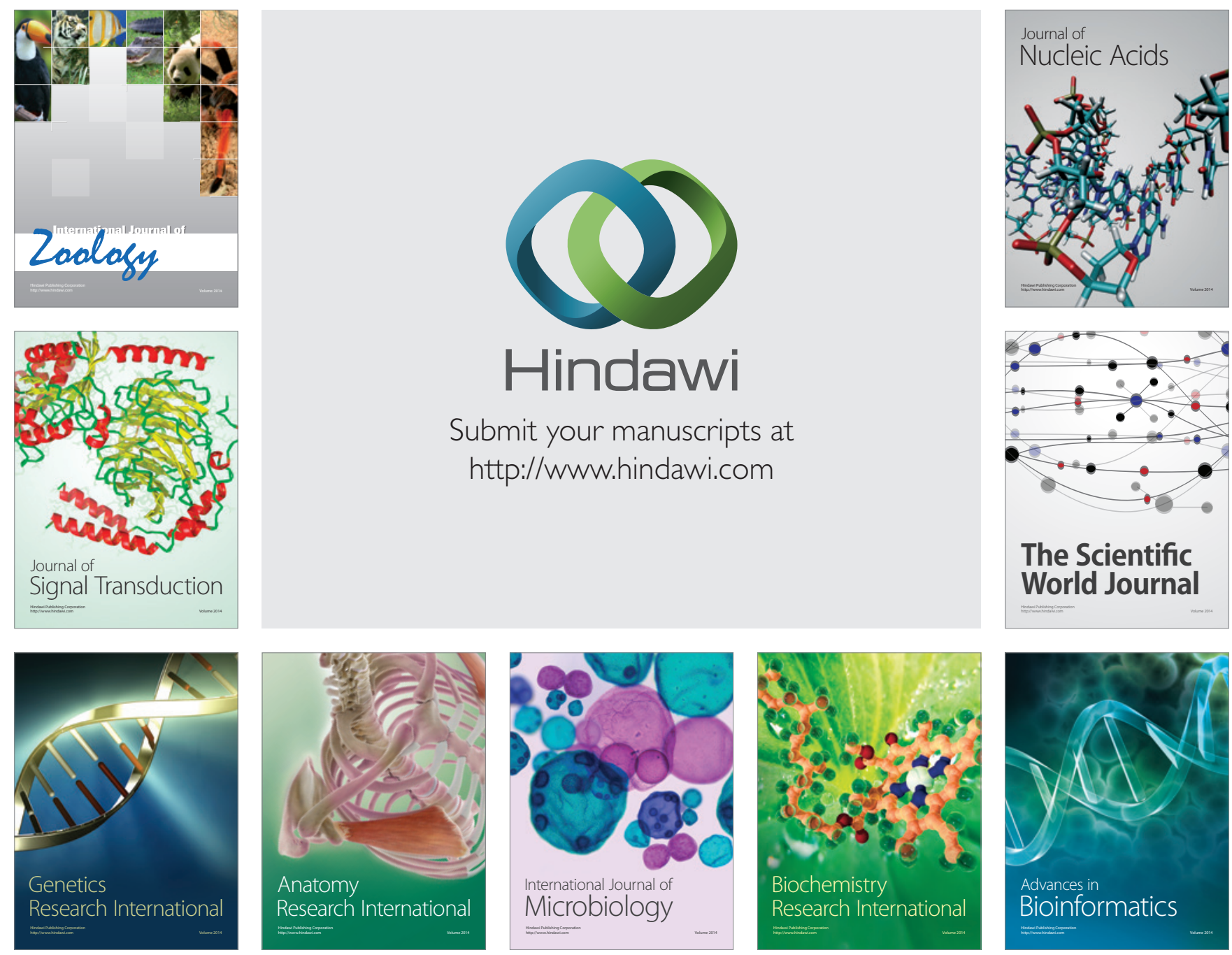

The Scientific World Journal
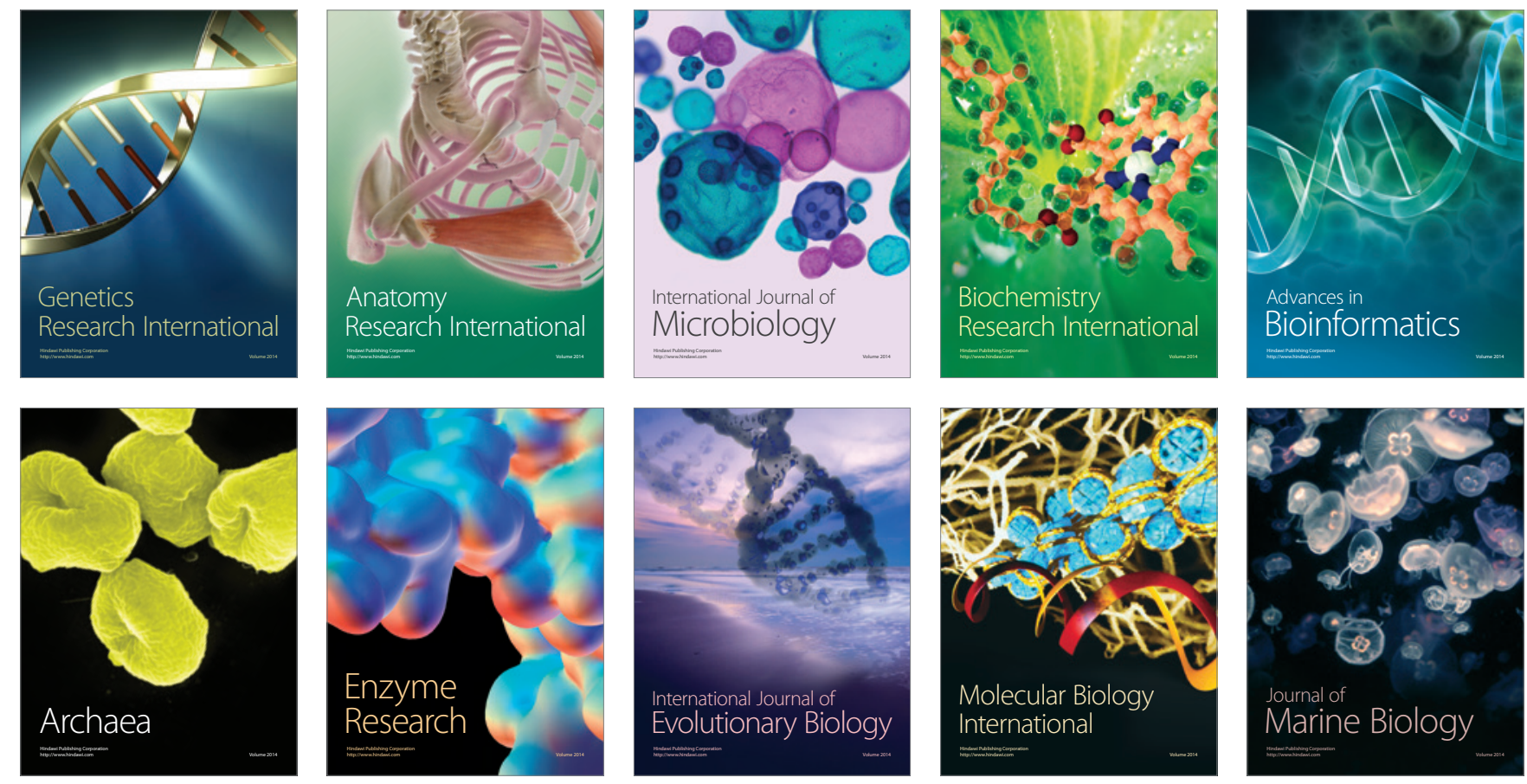